Research Article

\title{
Conservation Laws for Some Systems of Nonlinear Partial Differential Equations via Multiplier Approach
}

\author{
Rehana Naz \\ Centre for Mathematics and Statistical Sciences, Lahore School of Economics, Lahore 53200, Pakistan \\ Correspondence should be addressed to Rehana Naz, rehananaz_qau@yahoo.com
}

Received 26 July 2012; Accepted 20 September 2012

Academic Editor: Fazal M. Mahomed

Copyright (c) 2012 Rehana Naz. This is an open access article distributed under the Creative Commons Attribution License, which permits unrestricted use, distribution, and reproduction in any medium, provided the original work is properly cited.

The conservation laws for the integrable coupled KDV type system, complexly coupled kdv system, coupled system arising from complex-valued KDV in magnetized plasma, Ito integrable system, and Navier stokes equations of gas dynamics are computed by multipliers approach. First of all, we calculate the multipliers depending on dependent variables, independent variables, and derivatives of dependent variables up to some fixed order. The conservation laws fluxes are computed corresponding to each conserved vector. For all understudying systems, the local conservation laws are established by utilizing the multiplier approach.

\section{Introduction}

The partial differential equations, which arise in the sciences, dynamics, fluid mechanics, electromagnetism, economics and so forth, express conservation of mass, momentum, energy, electric charge, or value of firm. All the conservation laws of partial differential equations may not have physical interpretation but are essential in studying the integrability of the PDE. The high number of conservation laws for a partial differential equation grantees that the partial differential equation is strongly integrable and can be linearized or explicitly solved [1]. Moreover, the conservation laws are used for analysis, particularly, development of numerical schemes, soliton solutions, study of properties such as bi-Hamiltonian structures and recursion operators, and reduction of partial differential equations.

There are different methods for the construction of conservation laws as described by Naz [2], Naz et al. [3], Bluman et al. [4], Hereman et al. [5], and references therein. Rocha Filho and Figueiredo [6] developed computer packages based on Noether's method for the variational problems. Wolf [7] and Wolf et al. [8] introduced computer programmes in REDUCE to calculate conservation laws. 
In this work, the multiplier approach is used to derive the conservation laws for some systems of partial differential equations important due to physical point of view. Stuedel [9] introduced the multiplier approach and the conserved vectors were written in a characteristic form as $D_{i} T^{i}=\Lambda^{\alpha} E_{\alpha}$. The determining equations for the multipliers (characteristics) were obtained by taking the variational derivative of $D_{i} T^{i}=Q^{\alpha} E_{\alpha}$ for the arbitrary functions not only for solutions of system of partial differential equations [10]. A conserved vector is associated with each multiplier. The conservation laws for the integrable coupled kdv-type system, complexly coupled KDA system, coupled system arising from complex-valued KDV in magnetized plasma, Ito integrable system, and Navier stokes equations of gas dynamics are computed by utilizing the multiplier approach. The conserved vectors derived here can be used in constructing the solutions of underlying systems in the following different ways. The corresponding potential system can be written for the conservation laws, and symmetry reductions [11] can be carried out. Another approach to deduce exact solutions is via the double reduction theory [12-14]. The exact solution can be derived if the conservation laws give physical conserved quantities like Naz et al. [15]. The exact solutions of systems under consideration are subject of future work.

The outline of paper is as follows. In Section 2, some definitions related with multiplier approach are presented. The conservation laws for integrable coupled kdv-type system, complexly coupled kdv system, coupled system arising from complex-valued KDV in magnetized plasma, Ito integrable system, and Navier stokes equations of gas dynamics are constructed in Section 3. Conclusions are summarized in Section 4.

\section{Preliminaries}

Consider a $k$ th-order system of partial differential equations (PDEs) with $n$ independent variables $x \equiv\left(x^{1}, x^{2}, \ldots, x^{n}\right)$ and $m$ dependent variables $u \equiv\left(u^{1}, u^{2}, \ldots, u^{m}\right)$ defined as,

$$
E_{\alpha}\left(x, u, u_{(1)}, u_{(2)}, \ldots, u_{(k)}\right)=0, \quad \alpha=1,2, \ldots, m
$$

where $u_{(i)}$ is the collection of $i$ th-order partial derivatives of $u$.

(1) The Euler operator is defined by

$$
\frac{\delta}{\delta u^{\alpha}}=\frac{\partial}{\partial u^{\alpha}}-D_{i} \frac{\partial}{\partial u_{i}^{\alpha}}+D_{i} D_{j} \frac{\partial}{\partial u_{i j}^{\alpha}}-\cdots,
$$

where

$$
D_{i}=\frac{\partial}{\partial x^{i}}+u_{i}^{\alpha} \frac{\partial}{\partial u^{\alpha}}+u_{i j}^{\alpha} \frac{\partial}{\partial u_{j}^{\alpha}}+\cdots, \quad i=1,2
$$

is the total derivative operator with respect to $x^{i}$.

(2) A conserved vector of (2.1) is an $n$-tuple $T=\left(T^{1}, T^{2}, \ldots, T^{n}\right), T^{i} \in A, i=1,2 \ldots n$, such that

$$
D_{i} T^{i}=0
$$

holds for all solutions of (2.1). Equation (2.4) is called a local conservation law. 
(3) The multipliers $\Lambda^{\alpha}$ of system (2.1) has the property

$$
D_{i} T^{i}=\Lambda^{\alpha} E_{\alpha}
$$

for the arbitrary function $u^{\alpha}[9,10]$.

(4) The determining equations for the multipliers are obtained by taking variational derivative of (2.5) (see [10]):

$$
\frac{\delta}{\delta u^{\alpha}}\left[\Lambda^{\alpha} E_{\alpha}\right]=0
$$

Equation (2.6) holds for the arbitrary functions $u^{\alpha}$ not only for the solutions of system (2.1).

Equation (2.6) yields multipliers for all local conservation laws. Then conserved vectors can be derived systematically using (2.5) as the determining equation. But in some problems it is not difficult to construct the conserved vectors by elementary manipulations once the multiplier has been determined.

\section{Conservation Laws for Nonlinear Systems of Partial Differential Equations}

\subsection{Integrable Coupled System}

Consider the integrable coupled system $[16,17]$

$$
\begin{aligned}
& E_{1}=u_{t}+\left[u_{x x}-(k+3)(k+6) u^{2}-k^{2} v^{2}\right]_{x}+2 k\left[(k+6) v u_{x}+(k+3) u v_{x}=0\right. \\
& E_{2}=v_{t}+\left[v_{x x}-k(k-3) v^{2}-(k+3)^{2} u^{2}\right]_{x}+2(k+3)\left[k v u_{x}+(k-3) u v_{x}=0 .\right.
\end{aligned}
$$

The group invariant solution of (3.1) was derived in [17]. Here we will construct conservation laws for coupled system (3.1). Consider simple multipliers of the form $\Lambda_{1}(t, x, u, v)$ and $\Lambda_{2}(t, x, u, v)$. Multipliers $\Lambda_{1}$ and $\Lambda_{2}$ for the system (3.1) have the property that

$$
\Lambda_{1} E_{1}+\Lambda_{2} E_{2}=D_{t} T^{1}+D_{x} T^{2}
$$

for all functions $u(t, x)$ and $v(t, x)$ where the total derivative operators $D_{t}$ and $D_{x}$ from (2.3) are

$$
\begin{gathered}
D_{t}=\frac{\partial}{\partial t}+u_{t} \frac{\partial}{\partial u}+v_{t} \frac{\partial}{\partial v}+u_{t t} \frac{\partial}{\partial u_{t}}+v_{t t} \frac{\partial}{\partial v_{t}}+u_{t x} \frac{\partial}{\partial u_{x}}+v_{t x} \frac{\partial}{\partial v_{x}}+\cdots \\
D_{x}=\frac{\partial}{\partial x}+u_{x} \frac{\partial}{\partial u}+v_{x} \frac{\partial}{\partial v}+u_{x x} \frac{\partial}{\partial u_{x}}+v_{x x} \frac{\partial}{\partial v_{x}}+u_{x t} \frac{\partial}{\partial u_{t}}+v_{x t} \frac{\partial}{\partial v_{t}}+\cdots
\end{gathered}
$$

The right-hand side of (3.2) is a divergence expression and $T^{1}$ and $T^{2}$ are the components of the conserved vector $T=\left(T^{1}, T^{2}\right)$. The determining equations for the multipliers 
$\Lambda_{1}$ and $\Lambda_{2}$ are

$$
\begin{aligned}
& \frac{\delta}{\delta u}\left[\Lambda_{1} E_{1}+\Lambda_{2} E_{2}\right]=0, \\
& \frac{\delta}{\delta v}\left[\Lambda_{1} E_{1}+\Lambda_{2} E_{2}\right]=0
\end{aligned}
$$

where $\delta / \delta u$ and $\delta / \delta v$ are the standard Euler operators defined in (2.2), which annihilate divergence expressions:

$$
\begin{gathered}
\frac{\delta}{\delta u}=\frac{\partial}{\partial u}-D_{t} \frac{\partial}{\partial u_{t}}-D_{x} \frac{\partial}{\partial u_{x}}+D_{t}^{2} \frac{\partial}{\partial u_{t t}}+D_{t} D_{x} \frac{\partial}{\partial u_{t x}}+D_{x}^{2} \frac{\partial}{\partial u_{x x}}-\cdots \\
\frac{\delta}{\delta v}=\frac{\partial}{\partial v}-D_{t} \frac{\partial}{\partial v_{t}}-D_{x} \frac{\partial}{\partial v_{x}}+D_{t}^{2} \frac{\partial}{\partial v_{t t}}+D_{t} D_{x} \frac{\partial}{\partial v_{t x}}+D_{x}^{2} \frac{\partial}{\partial v_{x x}}-\cdots
\end{gathered}
$$

Separating (3.4) and (3.5), after expansion, with respect to different combinations of derivatives of $u$ and $v$, yields the following overdetermined system:

$$
\begin{gathered}
\Lambda_{2 x x}=0, \quad \Lambda_{2 v x}=0, \quad \Lambda_{2 v v}=0 \\
\Lambda_{1 t}=-\frac{12}{k}(k+3)[(u-v) k+3 u] \Lambda_{2 x}, \quad \Lambda_{1 x}=-\frac{(k+3)}{k} \Lambda_{2 x} \\
\Lambda_{2 t}=12[(k+3) u-k v] \Lambda_{2 x} \\
\Lambda_{1 u}=\frac{(k+3)\left[\left(2 k^{2}+6 k\right) \Lambda_{2}+2 \Lambda_{1} k^{2}+(k+3)((u-v) k+3 u)\right] \Lambda_{2 v}}{k^{2}[(u-v) k+3 u]} \\
\Lambda_{2 u}=\frac{\left(-3 k-k^{2}\right) \Lambda_{2}-\Lambda_{1} k^{2}-(k+3)((u-v) k+3 u) \Lambda_{2 v}}{[(u-v) k+3 u] k} \\
\Lambda_{1 v}=\frac{\left(-3 k-k^{2}\right) \Lambda_{2}-\Lambda_{1} k^{2}-(k+3)((u-v) k+3 u) \Lambda_{2 v}}{[(u-v) k+3 u] k}
\end{gathered}
$$

The solution of system (3.8) yields following four multipliers:

$$
\begin{gathered}
\Lambda_{1}^{(1)}=1, \quad \Lambda_{2}^{(1)}=-\frac{k+3}{k}, \\
\Lambda_{1}^{(2)}=-\frac{1}{k^{2}}(k+3)^{2} u, \quad \Lambda_{2}^{(2)}=v, \\
\Lambda_{1}^{(3)}=\frac{1}{k}[-k v+2(k+3) u], \quad \Lambda_{2}^{(3)}=u, \\
\Lambda_{1}^{(4)}=-\frac{k+3}{k}[x+12(k+3) u t-12 k v t], \\
\Lambda_{2}^{(4)}=x+12(k+3) u t-12 k t v .
\end{gathered}
$$


From (3.2) and (3.9), we obtained following four conserved vectors:

$$
\begin{aligned}
& T_{1}^{(1)}=-\frac{1}{k}[(k+3) u-k v], \\
& T_{2}^{(1)}=\frac{1}{k}\left[6(k+3)^{2} u^{2}-12 k(k+3) u v+k v_{x x}-k u_{x x}-3 u_{x x}\right], \\
& T_{1}^{(2)}=-\frac{1}{2 k^{2}}\left[u^{2}(k+3)^{2}-k^{2} v^{2}\right], \\
& T_{2}^{(2)}=\frac{1}{6 k^{2}}\left[3(k+3)^{2} u_{x}^{2}-3 k^{2} v_{x}^{2}+4(k+6)(k+3)^{3} u^{3}-4 k^{3}(k-3) v^{3}\right. \\
& \left.-12 k(k+3)^{3} u^{2} v+12 k^{3}(k+3) u v^{2}-6(k+3)^{2} u u_{x x}+6 k^{2} v v_{x x}\right], \\
& T_{1}^{(3)}=-\frac{1}{k}\left[(k+3) u^{2}-k u v\right], \\
& T_{2}^{(3)}=\frac{1}{3 k}\left[3(k+3) u_{x}^{2}+2(k+3)^{2}(k+12) u^{3}\right. \\
& -2 k^{3} v^{3}-6 k(k+3)(k+9) u^{2} v+6 k^{2}(k+6) u v^{2} \\
& \left.-6(k+3) u u_{x x}+3 k u_{x x} v+3 k u v_{x x}-3 k u_{x} v_{x}\right], \\
& T_{1}^{(4)}=-\frac{1}{k}\left[6(k+3)^{2} t u^{2}+6 k^{2} t v^{2}\right. \\
& \left.-12 k(k+3) t u v+(k+3) u_{x}-k v_{x}\right], \\
& T_{2}^{(4)}=\frac{1}{k}\left[-(k+3)(x+12(k+3) t u-12 k t v) u_{x x}\right. \\
& +(x+12(k+3) u t-12 k t v) k v_{x x}+6(k+3)^{2} t u_{x}^{2}+6 t k^{2} v_{x}^{2} \\
& -12 k(k+3) t u_{x} v_{x}+(k+3) u_{x}-k v_{x}+48(k+3)^{3} t u^{3} \\
& -48 k^{3} t v^{3}-144 k(k+3)^{2} t u^{2} v+144 k^{2}(k+3) t u v^{2} \\
& \left.+6(k+3)^{2} x u^{2}+6 k^{2} x v^{2}-12 k(k+3) x u v\right] \text {. }
\end{aligned}
$$

\subsection{Higher-Order Conservation Laws for Complexly Coupled KDV System}

The conservation laws of complexly coupled KDV were discussed in Naz [18]

$$
\begin{aligned}
& u_{t}-6 u u_{x}-6 v v_{x}-u_{x x x}=0 \\
& v_{t}-6 u v_{x}-6 v u_{x}-v_{x x x}=0
\end{aligned}
$$


and six conserved vectors were derived by multipliers approach with multipliers of form $\Lambda_{1}(t, x, u, v)$ and $\Lambda_{2}(t, x, u, v)$. Now we will consider higher-order multipliers and derive the associated conservation laws fluxes. The determining equations for multipliers of the form $\Lambda_{1}\left(t, x, u, v, u_{x}, v_{x}, u_{x x}, v_{x x}\right)$ and $\Lambda_{2}\left(t, x, u, v, u_{x}, v_{x}, u_{x x}, v_{x x}\right)$ from (2.6) are

$$
\begin{aligned}
& \frac{\delta}{\delta u}\left[\Lambda_{1}\left(u_{t}-6 u u_{x}-6 v v_{x}-u_{x x x}\right)+\Lambda_{2}\left(v_{t}-6 u v_{x}-6 v u_{x}-v_{x x x}\right)\right]=0 \\
& \frac{\delta}{\delta v}\left[\Lambda_{1}\left(u_{t}-6 u u_{x}-6 v v_{x}-u_{x x x}\right)+\Lambda_{2}\left(v_{t}-6 u v_{x}-6 v u_{x}-v_{x x x}\right)\right]=0
\end{aligned}
$$

where the standard Euler operators $\delta / \delta u$ and $\delta / \delta v$ are given by (3.6) and (3.7). Equations (3.12) and (3.13) are separated, after expansion, according to different combinations of derivatives of $u$ and $v$ and after some simplification the following system of equations for $\Lambda_{1}, \Lambda_{2}$ is obtained:

$$
\begin{aligned}
& \Lambda_{1 x x}=0, \quad \Lambda_{1 v x}=0, \quad \Lambda_{1 x v_{x x}}=0, \quad \Lambda_{1 v v_{x x}}=0, \quad \Lambda_{1 u_{x}}=0, \quad \Lambda_{1 v_{x}}=0, \\
& \Lambda_{2 x x}=0, \quad \Lambda_{2 v x}=0, \quad \Lambda_{2 x v_{x x}}=0, \quad \Lambda_{2 v v_{x x}}=0, \quad \Lambda_{2 u_{x}}=0, \quad \Lambda_{2 v_{x}}=0 \text {, } \\
& \Lambda_{1 v_{x x} v_{x x}}=0, \quad \Lambda_{2 v_{x x} v_{x x}}=0, \quad \Lambda_{2 v v}=6 \Lambda_{1 v_{x x}}, \quad \Lambda_{1 v v}=6 \Lambda_{2 v_{x x}}, \\
& \Lambda_{1 t}=6 \Lambda_{2 x} v+6 \Lambda_{1 x} u, \quad \Lambda_{2 t}=6 \Lambda_{1 x} v+6 \Lambda_{2 x} u, \\
& \Lambda_{1 u}=\Lambda_{2 v}, \quad \Lambda_{2 u}=\Lambda_{1 v}, \quad \Lambda_{1 u_{x x}}=\Lambda_{2 v_{x x}}, \quad \Lambda_{2 u_{x x}}=\Lambda_{1 v_{x x}} .
\end{aligned}
$$

The solution of system (3.14) yields

$$
\begin{aligned}
& \Lambda_{1}=c_{1}+u\left(c_{3}+c_{4} t\right)+v\left(c_{5}+c_{6} t\right)+\frac{x}{6} c_{4}+\left(3 u^{2}+3 v^{2}+u_{x x}\right) c_{7}+\left(6 u v+v_{x x}\right) c_{8}, \\
& \Lambda_{2}=c_{2}+v\left(c_{3}+c_{4} t\right)+u\left(c_{5}+c_{6} t\right)+\frac{x}{6} c_{6}+\left(6 u v+v_{x x}\right) c_{7}+\left(3 u^{2}+3 v^{2}+u_{x x}\right) c_{8},
\end{aligned}
$$

where $c_{1}, c_{2}, \ldots, c_{8}$ are arbitrary constants. The first six multipliers are same as derived in [18]. The two new multipliers are actually the higher-order multipliers associated with constants $c_{7}$ and $c_{8}$

$$
\begin{aligned}
& \Lambda_{1}^{(7)}=3 u^{2}+3 v^{2}+u_{x x}, \quad \Lambda_{2}^{(7)}=6 u v+v_{x x}, \\
& \Lambda_{1}^{(8)}=6 u v+v_{x x}, \quad \Lambda_{2}^{(8)}=3 u^{2}+3 v^{2}+u_{x x} .
\end{aligned}
$$


Equation (2.5) with multipliers given in (3.16) gives two new conservation laws

$$
\begin{aligned}
& T_{1}^{(7)}=3 u v^{2}+u^{3}+\frac{1}{2} u u_{x x}+\frac{1}{2} v v_{x x}, \\
& T_{2}^{(7)}=-6 u v v_{x x}-27 u^{2} v^{2}-\frac{1}{2} v v_{t x}-\frac{9}{2} u^{4}-\frac{1}{2} u_{x x}^{2} \\
& -3 u_{x x} v^{2}-3 u^{2} u_{x x}-\frac{9}{2} v^{4}-\frac{1}{2} v_{x x}^{2}-\frac{1}{2} u u_{t x}+\frac{1}{2} v_{x} v_{t}+\frac{1}{2} u_{x} u_{t} \\
& T_{1}^{(8)}=3 u^{2} v+v^{3}+\frac{1}{2} u v_{x x}+\frac{1}{2} u_{x x} v, \\
& T_{2}^{(8)}=-\frac{1}{2} u v_{t x}-18 u^{3} v-u_{x x} v_{x x}-18 u v^{3}-3 v^{2} v_{x x} \\
& -3 u^{2} v_{x x}-\frac{1}{2} v u_{t x}-6 u v u_{x x}+\frac{1}{2} u_{x} v_{t}+\frac{1}{2} v_{x} u_{t} .
\end{aligned}
$$

The two new higher-order conservation laws (3.17) are obtained for the system (3.11).

\subsection{Conservation Laws for Complex-Valued KDV in Magnetized Plasma}

The complexly coupled KDV

$$
w_{t}-\left(|w|^{2} w\right)_{x}-w_{x x x}=0
$$

arises in the study of the asymptotic investigation of electrostatic waves of a magnetized

plasma [19]. The variable $w$ is the complex field amplitude $w=u+i v$. The representation of (3.18) in real field variables $u$ and $v$ is

$$
\begin{aligned}
& u_{t}-3 u^{2} u_{x}-2 u v v_{x}-v^{2} u_{x}-u_{x x x}=0 \\
& v_{t}-3 v^{2} v_{x}-2 u v u_{x}-u^{2} v_{x}-v_{x x x}=0
\end{aligned}
$$

The conservation laws for system (3.19) are derived here by using multiplier approach. The determining equations for multipliers of the form $\Lambda_{1}\left(t, x, u, v, u_{x}, v_{x}, u_{x x}, v_{x x}\right)$ and $\Lambda_{2}\left(t, x, u, v, u_{x}, v_{x}, u_{x x}, v_{x x}\right)$ from (2.6) are

$$
\begin{aligned}
& \frac{\delta}{\delta u}\left[\Lambda_{1}\left(u_{t}-3 u^{2} u_{x}-2 u v v_{x}-v^{2} u_{x}-u_{x x x}\right)+\Lambda_{2}\left(v_{t}-3 v^{2} v_{x}-2 u v u_{x}-u^{2} v_{x}-v_{x x x}\right)\right]=0, \\
& \frac{\delta}{\delta v}\left[\Lambda_{1}\left(u_{t}-3 u^{2} u_{x}-2 u v v_{x}-v^{2} u_{x}-u_{x x x}\right)+\Lambda_{2}\left(v_{t}-3 v^{2} v_{x}-2 u v u_{x}-u^{2} v_{x}-v_{x x x}\right)\right]=0 .
\end{aligned}
$$


Equation (3.20) finally results in the following overdetermined system:

$$
\begin{aligned}
& \Lambda_{2 x x}=0, \quad \Lambda_{1 u_{x}}=0, \quad \Lambda_{2 u_{x}}=0, \quad \Lambda_{1 v_{x}}=0, \quad \Lambda_{2 v_{x}}=0, \\
& \Lambda_{1 u_{x x}}=\Lambda_{2 v_{x x}}, \quad \Lambda_{2 u_{x x}}=0, \quad \Lambda_{1 v_{x x}}=0, \\
& \Lambda_{1 u_{x x}}=\Lambda_{2 v_{x x}}, \quad \Lambda_{2 u_{x x}}=0, \quad \Lambda_{1 v_{x x}}=0, \\
& \Lambda_{2 v x}=\frac{\Lambda_{2 x}}{v}, \quad \Lambda_{2 x v_{x x}}=0, \quad \Lambda_{2 v v}=6 v \Lambda_{2 v_{x x}} \\
& \Lambda_{2 v v_{x x}}=0, \quad \Lambda_{2 v_{x x} v_{x x}}=0, \\
& \Lambda_{1 t}=\frac{3}{v}\left(u_{x x}+u^{3}+v^{2} u\right) \Lambda_{2 x} \\
& \Lambda_{2 t}=\frac{3}{v}\left(\Lambda_{2 x} v_{x x}+\Lambda_{2 x} u^{2} v+\Lambda_{2 x} v^{3}\right), \\
& \Lambda_{1 x}=\frac{\Lambda_{2 x} u}{v} \\
& \Lambda_{1 u}=2 \Lambda_{2 v_{x x}} u^{2}-2 \Lambda_{2 v_{x x}} v^{2}+\Lambda_{2 v} \\
& \Lambda_{2 u}=2 \Lambda_{2 v_{x x}} u v \text {, } \\
& \Lambda_{1 v}=2 \Lambda_{2 v_{x x}} u v .
\end{aligned}
$$

The solution of system (3.21) yields following five multipliers:

$$
\begin{gathered}
\Lambda_{1}^{(1)}=0, \quad \Lambda_{2}^{(1)}=1, \\
\Lambda_{1}^{(2)}=1, \quad \Lambda_{2}^{(2)}=0, \\
\Lambda_{1}^{(3)}=u, \quad \Lambda_{2}^{(3)}=v, \\
\Lambda_{1}^{(4)}=u^{3}+u v^{2}+u_{x x}, \\
\Lambda_{2}^{(4)}=u^{2} v+v^{3}+v_{x x}, \\
\Lambda_{1}^{(5)}=3 t\left(u^{3}+u v^{2}+u_{x x}\right)+x u, \\
\Lambda_{2}^{(5)}=3 t\left(v^{3}+u^{2} v+v_{x x}\right)+x v .
\end{gathered}
$$


Journal of Applied Mathematics

The corresponding conserved vectors conserved vectors are

$$
\begin{aligned}
& T_{1}^{(1)}=v, \quad T_{2}^{(1)}=-u^{2} v-v^{3}-v_{x x}, \\
& T_{1}^{(2)}=u, \quad T_{2}^{(2)}=-u^{3}-u v^{2}-u_{x x}, \\
& T_{1}^{(3)}=\frac{u^{2}}{2}+\frac{v^{2}}{2}, \\
& T_{2}^{(3)}=\frac{u_{x}^{2}}{2}+\frac{v_{x}^{2}}{2}-u u_{x x}-v v_{x x}-\frac{3}{4} u^{4}-\frac{3}{4} v^{4}-\frac{3}{2} u^{2} v^{2}, \\
& T_{1}^{(4)}=\frac{1}{4}\left[u^{4}+v^{4}+2 u^{2} v^{2}+2 u u_{x x}+2 v v_{x x}\right] \\
& T_{2}^{(4)}=-\frac{1}{2}\left[u^{6}+v^{6}+u_{x x}^{2}+v_{x x}^{2}+2 u v^{2} u_{x x}+2 u^{2} v v_{x x}-v_{x} v_{t}\right. \\
& \left.\quad-u_{x} u_{t}+2 u^{3} u_{x x}+3 u^{4} v^{2}+3 u^{2} v^{4}+2 v^{3} v_{x x}+v v_{t x}+u u_{t x}\right], \\
& T_{1}^{(5)}=\frac{3 t}{4}\left[u^{4}+v^{4}+2 u^{2} v^{2}+2 u u_{x x}+2 v v_{x x}\right]+\frac{x}{2} u^{2}+\frac{x}{2} v^{2}, \\
& T_{2}^{(5)}=-\frac{3 t}{2}\left[u^{6}+v^{6}+u_{x x}^{2}+v_{x x}^{2}+2 u v^{2} u_{x x}+2 u^{2} v v_{x x}-v_{x} v_{t}\right. \\
& \left.\quad-u_{x} u_{t}+2 u^{3} u_{x x}+3 u^{4} v^{2}+3 u^{2} v^{4}+2 v^{3} v_{x x}+v v_{t x}+u u_{t x}\right] \\
& -\frac{1}{2} u u_{x}-\frac{1}{2} v v_{x}+x\left[\frac{1}{2} u_{x}^{2}+\frac{1}{2} v_{x}^{2}-\frac{3}{4} u^{4}-\frac{3}{4} v^{4}-u u_{x x}-v v_{x x}-\frac{3}{2} u^{2} v^{2}\right] .
\end{aligned}
$$

\subsection{Conservation Laws for Ito Integrable System}

Consider the following integrable Ito coupled system [20]:

$$
\begin{gathered}
u_{t}=v_{x} \\
v_{t}=-2\left(v_{x x x}+3 u v_{x}+3 v u_{x}\right)-12 w w_{x}, \\
w_{t}=w_{x x x}+3 u w_{x}
\end{gathered}
$$

where $u(t, x), v(t, x)$ and $w(t, x)$. For simplicity, consider multipliers of the form $\Lambda_{1}=$ $\Lambda_{1}(t, x, u, v, w), \Lambda_{2}=\Lambda_{2}(t, x, u, v, w)$, and $\Lambda_{3}=\Lambda_{3}(t, x, u, v, w)$.

The determining equations for multipliers $\Lambda_{1}, \Lambda_{2}$, and $\Lambda_{3}$ from (2.6) are

$$
\begin{aligned}
& \frac{\delta}{\delta u}\left[\Lambda_{1}\left(u_{t}-v_{x}\right)+\Lambda_{2}\left(v_{t}+2 v_{x x x}+6 u v_{x}+6 v u_{x}+12 w w_{x}\right)+\Lambda_{3}\left(w_{t}-w_{x x x}-3 u w_{x}\right)\right]=0, \\
& \frac{\delta}{\delta v}\left[\Lambda_{1}\left(u_{t}-v_{x}\right)+\Lambda_{2}\left(v_{t}+2 v_{x x x}+6 u v_{x}+6 v u_{x}+12 w w_{x}\right)+\Lambda_{3}\left(w_{t}-w_{x x x}-3 u w_{x}\right)\right]=0, \\
& \frac{\delta}{\delta w}\left[\Lambda_{1}\left(u_{t}-v_{x}\right)+\Lambda_{2}\left(v_{t}+2 v_{x x x}+6 u v_{x}+6 v u_{x}+12 w w_{x}\right)+\Lambda_{3}\left(w_{t}-w_{x x x}-3 u w_{x}\right)\right]=0,
\end{aligned}
$$


where the standard Euler operators $\delta / \delta u, \delta / \delta v$, and $\delta / \delta w$ can be computed from (2.2). Separating (3.25)-(3.27), after expansion, according to different combinations of derivatives of $u, v$, and $w$ and after some simplification following system of equations for $\Lambda_{1}, \Lambda_{2}, \Lambda_{3}$ is obtained:

$$
\begin{aligned}
& \Lambda_{1 x x}=0, \quad \Lambda_{1 t}=0, \quad \Lambda_{1 u}=0, \quad \Lambda_{1 v}=0, \quad \Lambda_{1 w}=0, \\
& \Lambda_{2 x}=0, \quad \Lambda_{2 u}=0, \quad \Lambda_{2 v}=0, \quad \Lambda_{2 w}=0, \\
& \Lambda_{2 t}=\Lambda_{1 x}, \quad \Lambda_{3}(t, x, u, v, w)=0 .
\end{aligned}
$$

The system of determining equation (3.28) yields

$$
\Lambda_{1}=c_{1}+c_{2} x, \quad \Lambda_{2}=c_{3}+c_{2} t, \quad \Lambda_{3}=0,
$$

where $c_{1}, c_{2}, c_{3}$ are arbitrary constants and we have three multipliers

$$
\begin{array}{lll}
\Lambda_{1}^{(1)}=1, & \Lambda_{2}^{(1)}=0, & \Lambda_{3}^{(1)}=0, \\
\Lambda_{1}^{(2)}=x, & \Lambda_{2}^{(2)}=t, & \Lambda_{3}^{(2)}=0, \\
\Lambda_{1}^{(3)}=0, & \Lambda_{2}^{(3)}=1, & \Lambda_{3}^{(3)}=0 .
\end{array}
$$

From (2.5), the conservation laws associated with multipliers given in (3.30) are

$$
\begin{gathered}
T_{1}^{(1)}=u, \quad T_{2}^{(1)}=-v, \\
T_{1}^{(2)}=u x+v t, \quad T_{2}^{(2)}=6 u v t+6 w^{2} t-v x+2 t v_{x x} \\
T_{1}^{(3)}=v, \quad T_{2}^{(3)}=6 u v+6 w^{2}+2 v_{x x} .
\end{gathered}
$$

The multiplier approach gave three nontrivial conservation laws for Ito system (3.24).

\subsection{Conservation Laws Navier-Stokes Equations for the Compressible Flow of a Heat-Conducting, Viscous Fluid for the Ideal Gas Case}

The one-dimensional compressible flow of a heat-conducting, viscous fluid for the ideal gas case is represented by the Navier-stokes equations [21]

$$
\begin{gathered}
E_{1}=\rho_{t}+\rho_{x} u+\rho u_{x}=0, \\
E_{2}=u_{t}+u u_{x}+R \frac{\theta \rho_{x}}{\rho}+R \theta_{x}-\mu \frac{u_{x x}}{\rho}=0, \\
E_{3}=\theta_{t}+u \theta_{x}+\frac{R}{c_{v}} \theta u_{x}-\frac{\mu}{c_{v}} \frac{u_{x}^{2}}{\rho}-\frac{k}{c_{v}} \frac{\theta_{x x}}{\rho}=0,
\end{gathered}
$$


where $u(t, x), \rho(t, x)$, and $\theta(t, x)$ are the density, velocity, and temperature, $\mu>0$ is the viscosity coefficient, $k>0$ is the heat conductivity coefficient, $R>0$ is called the ideal gas constant, $c_{v}>0$ represents the specific heat at constant volume. Consider multipliers of the form $\Lambda_{1}=\Lambda_{1}(t, x, u, \rho, \theta), \Lambda_{2}=\Lambda_{2}(t, x, u, \rho, \theta)$, and $\Lambda_{3}=\Lambda_{3}(t, x, u, \rho, \theta)$. The determining equations for multipliers $\Lambda_{1}, \Lambda_{2}$, and $\Lambda_{3}$ from (2.6) are

$$
\begin{aligned}
& \frac{\delta}{\delta u}\left[\Lambda_{1} E_{1}+\Lambda_{2} E_{2}+\Lambda_{3} E_{3}\right]=0, \\
& \frac{\delta}{\delta \rho}\left[\Lambda_{1} E_{1}+\Lambda_{2} E_{2}+\Lambda_{3} E_{3}\right]=0, \\
& \frac{\delta}{\delta \theta}\left[\Lambda_{1} E_{1}+\Lambda_{2} E_{2}+\Lambda_{3} E_{3}\right]=0,
\end{aligned}
$$

where the standard Euler operators $\delta / \delta u, \delta / \delta \rho$, and $\delta / \delta \theta$ can be calculated from (2.2). Expanding (3.33) and then separating according to different combinations of derivatives of $u, \rho$, and $\theta$ and after some simplification following system of equations for $\Lambda_{1}, \Lambda_{2}, \Lambda_{3}$ is obtained:

$$
\begin{gathered}
\Lambda_{1 x x}=0, \quad \Lambda_{1 t}=-u \Lambda_{1 x}, \quad \Lambda_{2 t}=-\Lambda_{1 x} \rho, \\
\Lambda_{3 t}=0, \quad \Lambda_{2 x}=0, \quad \Lambda_{3 x}=0, \quad \Lambda_{1 u}=\frac{\Lambda_{2}}{\rho}, \\
\Lambda_{2 u}=\frac{\Lambda_{3}}{c}, \quad \Lambda_{3 u}=0, \quad \Lambda_{1} \rho=0, \quad \Lambda_{2 \rho}=\frac{\Lambda_{2}}{\rho}, \\
\Lambda_{3 \rho}=\frac{\Lambda_{3}}{\rho}, \quad \Lambda_{1 \theta}=\frac{\Lambda 3}{\rho}, \quad \Lambda_{2 \theta}=0, \quad \Lambda_{3 \theta}=0, \\
R+c_{v} \neq 0, \quad-R+2 c_{v} \neq 0 .
\end{gathered}
$$

The adiabatic constant $\gamma=1+R / c_{v}$ has physical applications in region (1, (5/3)] (see e.g., [21, 22]). If $R+c_{v}=0$ or $-R+2 c_{v}=0$ then the value of $\gamma$ will be outside the region of physical significance. The assumptions $R+c_{v} \neq 0$ and $-R+2 c_{v} \neq 0$ are necessary for having a true physical model. The solution of system (3.34) results in the following four multipliers:

$$
\begin{array}{cc}
\Lambda_{1}^{(1)}=1, \quad \Lambda_{2}^{(1)}=0, \quad \Lambda_{3}^{(1)}=0, \\
\Lambda_{1}^{(2)}=u, \quad \Lambda_{2}^{(2)}=\rho, \quad \Lambda_{3}^{(2)}=0, \\
\Lambda_{1}^{(3)}=x-t u, \quad \Lambda_{2}^{(3)}=-t \rho, \quad \Lambda_{3}^{(3)}=0, \\
\Lambda_{1}^{(4)}=\theta+\frac{u^{2}}{2 c_{v}}, \quad \Lambda_{2}^{(4)}=\frac{u \rho}{c_{v}}, \quad \Lambda_{3}^{(4)}=\rho .
\end{array}
$$


The expressions for the fluxes are

$$
\begin{gathered}
T_{1}^{(1)}=\rho, \quad T_{2}^{(1)}=u \rho, \\
T_{1}^{(2)}=u \rho, \quad T_{2}^{(2)}=u^{2} \rho-\mu u_{x}+R \rho \theta, \\
T_{1}^{(3)}=\rho(x-t u), \quad T_{2}^{(3)}=x u \rho-t u^{2} \rho+\mu t u_{x}-R t \rho \theta, \\
T_{1}^{(4)}=\rho \theta+\frac{u^{2} \rho}{2 c_{v}}, \quad T_{2}^{(4)}=\frac{1}{2 c_{v}}\left[2 c_{v} u \rho \theta+u^{3} \rho+2 R u \rho \theta-2 \mu u u_{x}-2 k \theta_{x}\right] .
\end{gathered}
$$

We will get same results if we consider multipliers of the form $\Lambda_{i}=\Lambda_{i}\left(t, x, u, \rho, \theta, u_{x}\right.$, $\left.\rho_{x}, \theta_{x}\right), i=1,2,3$ or $\Lambda_{i}=\Lambda_{i}\left(t, x, u, \rho, \theta, u_{t}, \rho_{t}, \theta_{t}\right), i=1,2,3$.

\section{Conclusions}

The conservation laws for the integrable coupled kdv type system, complexly coupled $\mathrm{kdv}$ system, coupled system arising from complex-valued KDV in magnetized plasma, Ito integrable system, and Navier stokes equations of gas dynamics were computed by multipliers approach. The multipliers having dependence on dependent variables, independent variables, and derivatives of dependent variables up to some fixed order were constructed. After computing multipliers, the conservation laws fluxes were derived.

First of all, we considered integrable coupled kdv-type system and multiplier approach yielded four local conserved vectors. For the complexly coupled KDV system, total eight multipliers were obtained. The two new conserved vectors corresponding to second-order multipliers were obtained and were not found in [18]. The multiplier approach on coupled system arising from complex-valued KDV in magnetized plasma gave five conserved vectors. For Ito integrable system three and for Navier stokes equations of gas dynamics four, nontrivial conserved vectors were derived.

The conserved vectors derived here can be used in constructing the solutions of underlying PDE systems and will be considered in the future work.

\section{References}

[1] M. J. Ablowitz and P. A. Clarkson, Solitons, Nonlinear Evolution Equations and Inverse Scattering, vol. 149 of London Mathematical Society Lecture Note Series, Cambridge University Press, Cambridge, UK, 1991.

[2] R. Naz, Symmetry solutions and conservation laws for some partial differential equations in fluid mechanics [Ph.D. dissertation], University of the Witwatersrand, Johannesburg, South Africa, 2008.

[3] R. Naz, F. M. Mahomed, and D. P. Mason, "Comparison of different approaches to conservation laws for some partial differential equations in fluid mechanics," Applied Mathematics and Computation, vol. 205, no. 1, pp. 212-230, 2008.

[4] G. W. Bluman, A. F. Cheviakov, and S. C. Anco, Applications of Symmetry Methods to Partial Differential Equations, vol. 168 of Applied Mathematical Sciences, Springer, New York, 2010.

[5] W. Hereman, M. Colagrosso, R. Sayers et al., "Continuous and discrete homotopy operators and the computation of conservation laws," in Differential Equations with Symbolic Computation, Trends in Mathematics, pp. 255-290, Birkhäuser, Basel, Switzerland, 2005.

[6] T. M. Rocha Filho and A. Figueiredo, "[SADE] a Maple package for the symmetry analysis of differential equations," Computer Physics Communications, vol. 182, no. 2, pp. 467-476, 2011. 
[7] T. Wolf, "A comparison of four approaches to the calculation of conservation laws," European Journal of Applied Mathematics, vol. 13, no. 2, pp. 129-152, 2002.

[8] T. Wolf, A. Brand, and M. Mohammadzadeh, "Computer algebra algorithms and routines for the computation of conservation laws and fixing of gauge in differential expressions," Journal of Symbolic Computation, vol. 27, no. 2, pp. 221-238, 1999.

[9] H. Steudel, "Über die Zuordnung zwischen Invarianzeigenschaften und Erhaltungssätzen," Zeitschrift für Naturforschung, vol. 17, pp. 129-132, 1962.

[10] P. J. Olver, Applications of Lie Groups to Differential Equations, vol. 107 of Graduate Texts in Mathematics, Springer, New York, NY,USA, 2nd edition, 1993.

[11] G. Bluman, "Potential symmetries and equivalent conservation laws," in Modern Group Analysis: Advanced Analytical and Computational Methods in Mathematical Physics, pp. 71-84, Kluwer Academic, Dodrecht, The Netherlands, 1993.

[12] A. Sjöberg, "Double reduction of PDEs from the association of symmetries with conservation laws with applications," Applied Mathematics and Computation, vol. 184, no. 2, pp. 608-616, 2007.

[13] A. Sjöberg, "On double reductions from symmetries and conservation laws," Nonlinear Analysis: Real World Applications, vol. 10, no. 6, pp. 3472-3477, 2009.

[14] A. H. Bokhari, A. Y. Al-Dweik, F. D. Zaman, A. H. Kara, and F. M. Mahomed, "Generalization of the double reduction theory," Nonlinear Analysis: Real World Applications, vol. 11, no. 5, pp. 3763-3769, 2010.

[15] R. Naz, F. M. Mahomed, and D. P. Mason, "Conservation laws via the partial Lagrangian and group invariant solutions for radial and two-dimensional free jets," Nonlinear Analysis: Real World Applications, vol. 10, no. 6, pp. 3457-3465, 2009.

[16] S. Y. Lou, B. Tong, H.-c. Hu, and X.-y. Tang, "Coupled KdV equations derived from two-layer fluids," Journal of Physics A, vol. 39, no. 3, pp. 513-527, 2006.

[17] S. Qian and L. Tian, "Group-invariant solutions of a integrable coupled system," Nonlinear Analysis: Real World Applications, vol. 9, no. 4, pp. 1756-1767, 2008.

[18] R. Naz, "Conservation laws for a complexly coupled KdV system, coupled Burgers' system and Drinfeld-Sokolov-Wilson system via multiplier approach," Communications in Nonlinear Science and Numerical Simulation, vol. 15, no. 5, pp. 1177-1182, 2010.

[19] A. A. Mohammad and M. Can, "Exact solutions of the complex modified Korteweg-de Vries equation," Journal of Physics A, vol. 28, no. 11, pp. 3223-3233, 1995.

[20] H. A. Zedan, "Symmetry analysis of an integrable Ito coupled system," Computers \& Mathematics with Applications, vol. 60, no. 12, pp. 3088-3097, 2010.

[21] J. Wang, "Boundary layers for compressible Navier-Stokes equations with outflow boundary condition," Journal of Differential Equations, vol. 248, no. 5, pp. 1143-1174, 2010.

[22] A. N. W. Hone, "Painleve tests, singularity structure and integrability," Nonlinear Science, pp. 1-34, 2005. 


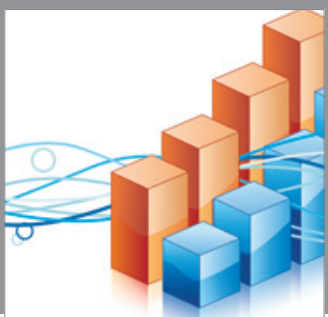

Advances in

Operations Research

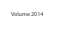

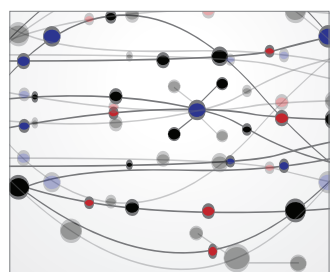

\section{The Scientific} World Journal
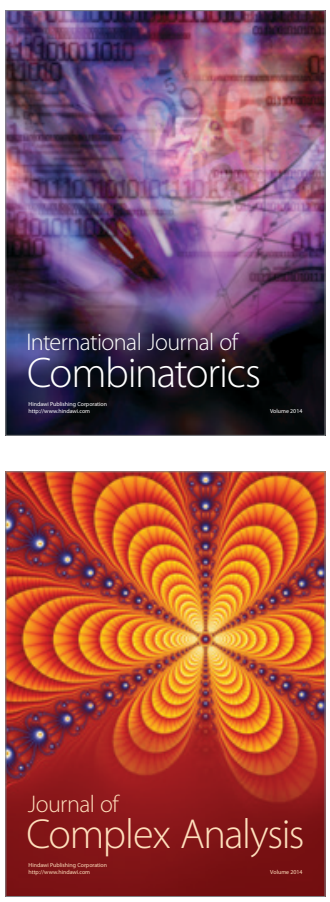

International Journal of

Mathematics and

Mathematical

Sciences
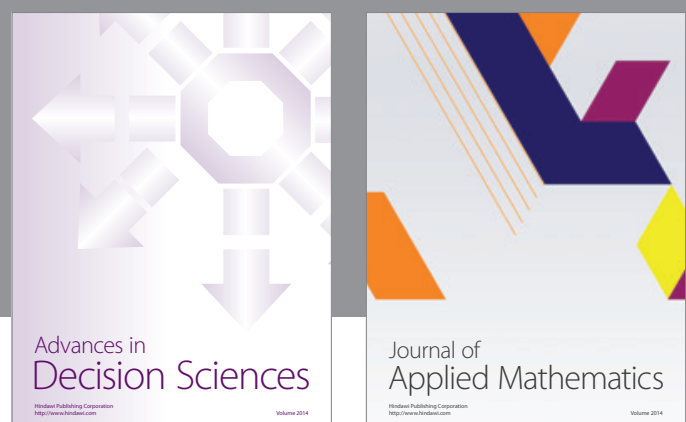

Journal of

Applied Mathematics
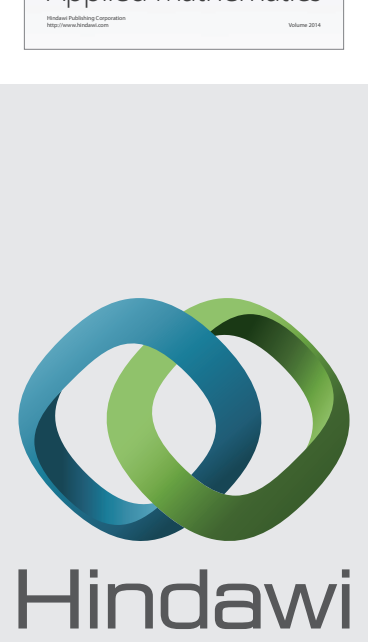

Submit your manuscripts at http://www.hindawi.com
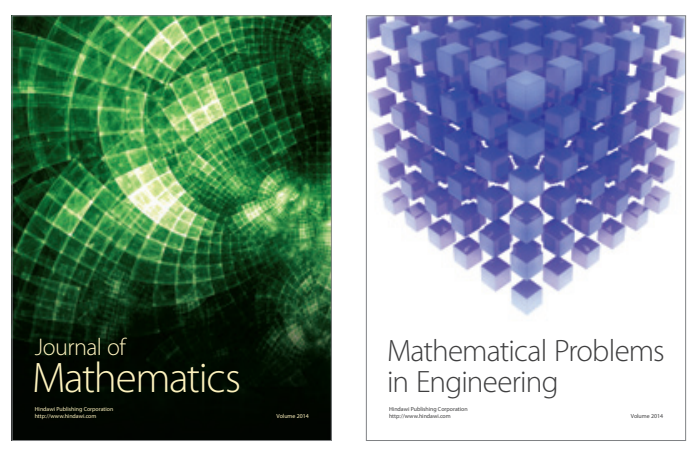

Mathematical Problems in Engineering
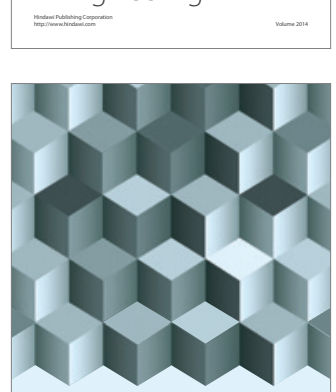

Journal of

Function Spaces
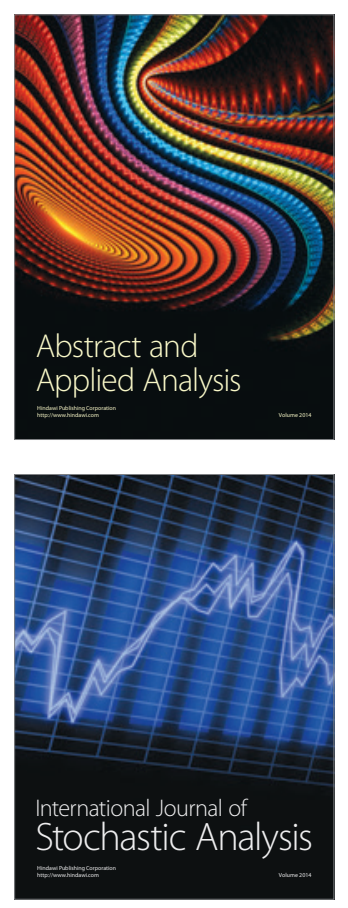

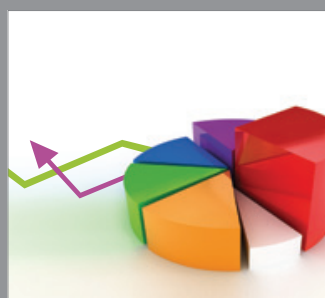

ournal of

Probability and Statistics

Promensencen
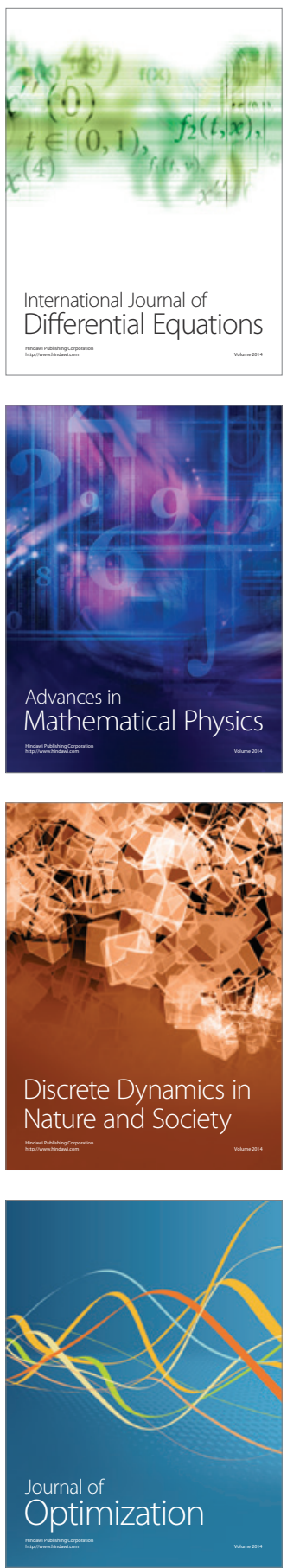